\title{
Heterogeneous Relay Selection
}

\author{
Mohamed Abouelseoud, Member, IEEE, and Aria Nosratinia, Fellow, IEEE
}

\begin{abstract}
Relay selection, a simple and efficient method of operating multi-relay networks, has in the past been considered only when all relays follow the same protocol. This work studies relay selection in heterogeneous relay networks where relays with different protocols can co-exist. The new techniques for the analysis of such networks are demonstrated on a mixture of decode-forward relays and amplify-forward relays, as well as a mixture of non-orthogonal amplify-forward (NAF) and dynamic decode-forward (DDF) relays, and also a mixture of NAF and compress-forward relays. In each case the diversity-multiplexing tradeoff is calculated. In the process, a noteworthy limitation of previous relay selection schemes is also relaxed and removed: In the past in multi-relay networks the direct source-destination link has been ignored whenever it introduces dependencies among selection decision variables, as it often does. This has been motivated chiefly by the lack of suitable analytical techniques for order statistics of dependent random variables. In this work, relay selection analysis is extended to allow the presence of a direct source-destination link. As a side benefit, the relay selection results in homogeneous networks (where relays are all of the same type) are also extended to include the presence of a sourcedestination link.
\end{abstract}

Index Terms-Relay, multiuser diversity, opportunistic communication, heterogeneous.

\section{INTRODUCTION}

$\mathbf{I}$ $\mathrm{N}$ a multi-relay scenario, relay selection can harvest diversity with relatively modest requirements compared with alternatives such as distributed space-time codes or distributed beamforming. An early example of cooperative selection appeared in [1]. Bletsas et al [2] investigated amplify-andforward (AF) relay selection, followed by many other works including [3], [4], [5]. Decode-and-forward (DF) relay selection has also received attention, e.g. [6] and many others [7], [6], [8]. The diversity multiplexing tradeoff (DMT) for relay selection has been investigated in [9] for addressing the multiplexing loss of DF relaying, and [10] for a combination of antenna selection and AF relay selection.

This work studies the performance of relay selection in heterogeneous relay networks where relays with different protocols co-exist. For example, a heterogeneous network may contain both DF relays and AF relays, or in the non-orthogonal transmission mode, the network may include dynamic decodeforward (DDF) relays and non-orthogonal amplify-forward (NAF) relays.

In the process of developing heterogeneous relay selection results, this paper also relaxes and removes a long-standing restriction on relay selection networks: In relay selection

Manuscript received May 14, 2012; revised September 11, 2012; accepted November 12, 2012. The associate editor coordinating the review of this paper and approving it for publication was Y. Jing.

The authors are with the University of Texas at Dallas, Richardson, TX 75080 USA (e-mail: \{m.abolsoud, aria\}@utdallas.edu).

This work was presented in part in Globecom 2010 and Asilomar 2010. Digital Object Identifier 10.1109/TWC.2013.013013.121689 the source-destination link introduces dependencies among decision variables ${ }^{1}$ and because of that previous works on relay selection often assumed the direct link to be non-existant, i.e., they assumed a two-hop geometry. However, ignoring the direct link is in general wasteful, and in particular interferes with the operation of compress-forward (CF), one of the more powerful relaying protocols. ${ }^{2}$ So there is ample motivation to produce analysis that can account for the direct link.

This paper studies a heterogeneous network (Figure 1) where one relay is selected for each transmission interval. In particular, the following combinations are studied in this paper: mixture of DF relays and AF relays, as well as a mixture of NAF relays and DDF relays, and also a mixture of NAF relays and CF relays. The diversity-multiplexing tradeoff of heterogeneous relay selection is analyzed in each of these cases.

A brief overview of our results is as follows: in a network of $n$ relays of the same type in the presence of a direct sourcedestination link, the orthogonal relaying protocols achieve the same DMT as non-orthogonal amplify-forward. At low multiplexing gains the dynamic decode-forward achieves the MISO upper bound. For high multiplexing gains, CF relay selection outperforms both DDF and NAF. DDF and CF relay selection outperform NAF at all multiplexing gains.

We observe that the DMT of heterogeneous orthogonal relaying is similar to the DMT of the corresponding homogeneous AF or DF relay systems. For heterogeneous nonorthogonal relaying we consider NAF, DDF, and CF. Relay selection among NAF and DDF relays gives maximal performance at high multiplexing gains, while providing diversity somewhere between that of NAF and DDF at low multiplexing gains. Relay selection among $\mathrm{CF}$ and NAF relays achieves a diversity that scales with the number of relays at high multiplexing gain as opposed to the fixed diversity for NAF.

An interesting direction that on first sight may seem related to this paper is hybrid relaying, where a relay adaptively changes its relaying protocol (e.g. AF/DF) [11], [12], [13], [14]. This class of work describes a new type of relay that obeys a complex decoding law. But this is distinct from a system containing multiple relays of different kind, which we call a heterogeneous system. True heterogeneous schemes have appeared very rarely in the literature: Jeong et al. [15] studied a system with multiple AF and DF relays without a direct link. Lusina et al. [16] presented a variation of the slotted amplify-forward (SAF) scheme of Yang and Belfiore [17] with two relays, where one of the relays is AF while the other (unlike [17]) is DF.

\footnotetext{
${ }^{1}$ With rare exceptions, e.g. [9]

${ }^{2}$ With no direct link and one selected relay, the destination is in outage if the relay cannot decode (due to cutset bound). So compress-forward is not particularly useful in a two-hop network.
} 


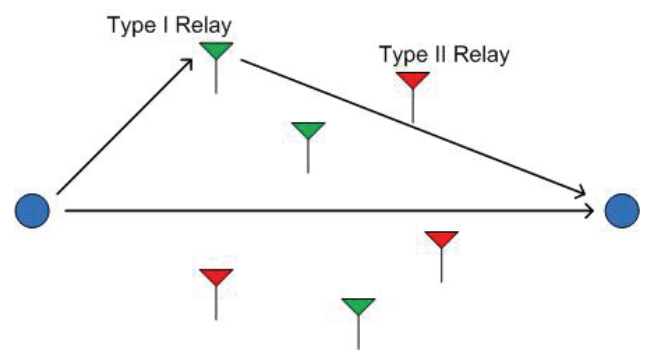

Fig. 1. Multi-relay channel with direct link, relays can be of different types.

\section{SySTEM Model}

We study a system of one source, one destination and $n$ relays, all half-duplex single-antenna nodes. There is a viable link between the source and destination. The destination can receive data from the source and the relays. Wireless links experience flat, quasi-static block fading. The channel coefficients $h_{s i}, h_{i d}$ and $h_{s d}$ represent the source-relay and relay-destination for relay $i$, and the source-destination link, respectively. The channel coefficients are independent and identically distributed circularly symmetric complex Gaussian random variables. ${ }^{3} n_{i} \sim \mathcal{C N}(0,1)$ and $n_{d} \sim \mathcal{C N}(0,1)$ are additive white Gaussian noise (AWGN) at relay $i$ and the receiver, respectively.

A block fading model is used where each message is transmitted within one coherence interval, which is sufficiently long to support codewords that allow standard coding arguments to be used. The relays and the destination have receive-side channel state information (CSI) and for the selection process, we assume all the CSIs are available. We use the framework of the diversity-multiplexing tradeoff [18]. The multiplexing gain $r$ and the diversity gain $d$ are defined as

$$
r=\lim _{\rho \rightarrow \infty} \frac{R(\rho)}{\log \rho}, \quad d=-\lim _{\rho \rightarrow \infty} \frac{\log P_{e}(\rho)}{\log \rho},
$$

where $\rho$ is the transmission SNR, $R(\rho)$ is the transmission rate and $P_{e}(\rho)$ is the error probability. The function $f(\rho)$ is said to be exponentially equal to $\rho^{v}$, denoted by $f(\rho) \doteq \rho^{v}$, if

$$
\lim _{\rho \rightarrow \infty} \frac{\log (f(\rho))}{\log (\rho)}=v
$$

\section{DMT For OpPortunistic Mode SElection}

We borrow the following results from [19], which apply to a general network with potentially multiple sources, destinations, and relays. An access mode is a communication session involving a subset of network nodes and selection is defined here by switching opportunistically between the available defined access modes. In this case, each relay together with the source and destination constitutes an access mode.

Theorem 1: [19] An upper bound for the DMT of a system opportunistically switches between $n$ wireless access modes is given by

$$
d(r) \leq d_{1}(r)+d_{2}^{\prime}(r)+\ldots+d_{n}^{\prime}(r),
$$

\footnotetext{
${ }^{3}$ For non-identically distributed channel coefficient, the same technique used in the paper can be applied. The assumption of identical distributions is made to make the results simpler to analyze
}

where $d_{1}(r)$ is the DMT of mode 1 and $d_{i}^{\prime}(r)$ is defined as

$$
d_{i}^{\prime}(r)=-\lim _{\rho \rightarrow \infty} \frac{\log \mathbb{P}\left(e_{i} \mid e_{i-1}, \ldots, e_{1}\right)}{\log \rho},
$$

for $i \in\{2, \ldots, n\}$ and $\mathbb{P}\left(e_{i} \mid e_{i-1}, \ldots, e_{1}\right)$ is the probability of error for the $i^{t h}$ mode of access given that all the previous modes of access are already in error.

At high SNR and with sufficiently long codewords, the error event is dominated by the outage event [18]. Thus, throughout this paper for simplicity the diversities are expressed in terms of outage events instead of error events.

Under certain conditions the bound in (3) is tight [19]. We have

$$
d(r)=d_{1}(r)+d_{2}^{\prime}(r)+\ldots+d_{n}^{\prime}(r),
$$

if the following two conditions are asymptotically satisfied:

1) Overall outage occurs only when all access modes are in outage.

2) Each access mode uses a codebook that achieve its individual (conditional) DMT.

The analysis in this paper uses instantaneous mutual information of each access mode for selection decisions, therefore the first condition is satisfied, and we assume proper codebooks are used to satisfy the second condition.

\section{Opportunistic Relay SElection}

We study a system of one source, one destination and $n$ relays. A relay is allowed to opportunistically access the channel and assist the source by transmitting to the destination. This generates various ways to access the channel (access modes), as seen in Figure 2. The source and destination can communicate without a relay, as shown in the first access mode, or a relay can forward a version of the signal received from the source. Since the source-destination link is shared among the relays, the selection process is much more complicated than the two-hop variety. More importantly, the shared link introduces dependency between the decision variables and this complicates the analysis. Theorem 1 provides a technique to analyze such a system by providing a method to combine the conditional DMTs of each access mode to produce the overall DMT. Naturally this is useful only if the conditional DMTs are tractable.

\section{A. Orthogonal Relaying}

A system is considered with $n$ orthogonal relays that are AF, $\mathrm{DF}$, or a combination. The selection criterion is as follows. If the direct link alone can support the transmission rate, no relay will be selected. Otherwise, the transmission will be assisted by a single selected relay, in which case the source transmits for half the transmission interval and the relay transmits for the second half of the transmission interval.

This creates $n+1$ access modes, where $n$ is the total number of relays in the system (Figure 2). The first access mode, the non-relayed access mode, is relay-free. The remaining relayassisted access modes each involves a relay in the usual twointerval orthogonal relay framework. Some of these access modes may be AF and some of them may be DF. Each will 

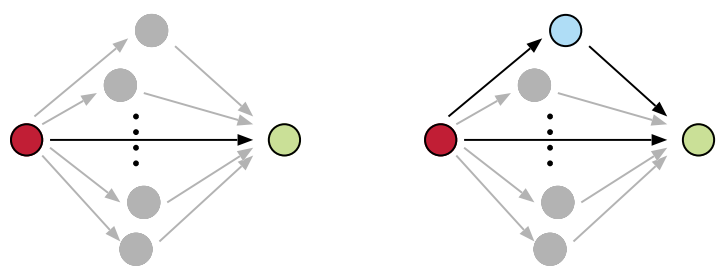

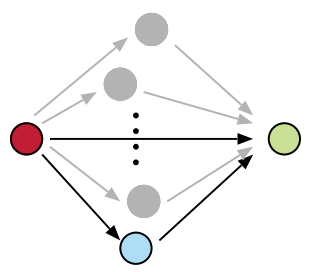

Fig. 2. The non-orthogonal access modes.

have an end-to-end mutual information calculated according to its own protocol.

1) Upper bound: Assume a genie provides the relays with a noise-free, error-free copy of the source message. Thus the source-relay channel is removed from the picture (effectively replaced with a perfect wire), and each selected relay together with the source will constitute a $2 \times 1$ MISO channel (Figure 3). Recall that there are $n$ different ways to select a relay, each corresponding to a relay-assisted mode, all of them sharing one link: the source destination link. The non-relayed mode is not affected by the genie.

Theorem 2: An upper bound for the opportunistic parallel relay channel with $n$ orthogonal relays is

$$
d(r)=(n+1)(1-r)^{+} .
$$

Proof: Using Theorem 1 under the tightness conditions guaranteeing Equation (5), the genie upper bound DMT is

$$
d(r)=d_{0}(r)+d_{1}^{\prime}(r)+\ldots+d_{n}^{\prime}(r),
$$

where $d_{0}(r)$ is the DMT of the non-relay mode (denoted Mode 0 ). This mode consists of a simple direct link whose DMT is $d_{0}(r)=(1-r)^{+}$and $d_{i}^{\prime}(r), i=1, \ldots, n$, are the conditional DMTs of the relay-assisted modes that are given by Equation (4).

For the first relay-assisted access mode (denoted Mode 1), the DMT is given by

$$
d_{1}^{\prime}(r)=-\lim _{\rho \rightarrow \infty} \frac{\log \mathbb{P}\left(\mathcal{O}_{1} \mid \mathcal{O}_{0}\right)}{\log \rho},
$$

where $\mathcal{O}_{i}$ represents the outage event for Mode $i$. The outage probability of Mode 1 given that Mode 0 is in outage

$$
\begin{gathered}
\mathbb{P}\left(\mathcal{O}_{1} \mid \mathcal{O}_{0}\right)=\mathbb{P}\left(\left\{\log \left(1+\left(\left|h_{s d}\right|^{2}+\left|h_{1 d}\right|^{2}\right) \rho\right)<r \log \rho\right\} \mid\right. \\
\left.\left\{\log \left(1+\left|h_{s d}\right|^{2} \rho\right)<r \log \rho\right\}\right) \\
=\mathbb{P}\left(\left\{\left|h_{s d}\right|^{2}+\left|h_{1 d}\right|^{2}<\frac{\rho^{r}-1}{\rho}\right\} \mid\left\{\left|h_{s d}\right|^{2}<\frac{\rho^{r}-1}{\rho}\right\}\right),
\end{gathered}
$$

where Mode 1 is equivalent to a $2 \times 1$ MISO channel and the conditioning means that one of its links is not capable of supporting the whole transmission rate. Using results from the Appendix and defining $\alpha=g_{1}(r, \rho)=\frac{\rho^{r}-1}{\rho}$, the conditional outage probability can be calculated as follows

$$
\begin{aligned}
& \mathbb{P}\left(\mathcal{O}_{1} \mid \mathcal{O}_{0}\right) \\
& =\int_{0}^{g_{1}(r, \rho)} f_{Z \mid B}(z) d z=\int_{0}^{g_{1}(r, \rho)} \frac{z e^{-z}}{1-e^{g_{1}(r, \rho)}} d z \\
& \doteq \frac{1-e^{-\rho^{r-1}}-\rho^{r-1} e^{-\rho^{r-1}}}{1-e^{-\rho^{r-1}}} \doteq \rho^{r-1} .
\end{aligned}
$$

Hence, $d_{1}^{\prime}(r)=(1-r)^{+}$. For Modes $i>1$, one can show that

$$
\begin{aligned}
& \mathbb{P}\left(\mathcal{O}_{i} \mid \mathcal{O}_{0}, \ldots, \mathcal{O}_{i-1}\right) \\
& \doteq \mathbb{P}\left(\left|h_{s d}\right|^{2}+\left|h_{i d}\right|^{2}<\rho^{r-1} \mid \mathcal{O}_{0}, \ldots, \mathcal{O}_{i-1}\right) \\
& \dot{\leq} \mathbb{P}\left(\left|h_{i d}\right|^{2}<\rho^{r-1} \mid \mathcal{O}_{0}, \ldots, \mathcal{O}_{i-1}\right)=\mathbb{P}\left(\left|h_{i d}\right|^{2}<\rho^{r-1}\right) .
\end{aligned}
$$

At the same time, $\mathbb{P}\left(\mathcal{O}_{i} \mid \mathcal{O}_{0}, \ldots, \mathcal{O}_{i-1}\right)$ is lower bounded by $\mathbb{P}\left(\mathcal{O}_{i} \mid \mathcal{O}_{0}\right)$, which can be calculated using the same technique used for $\mathbb{P}\left(\mathcal{O}_{1} \mid \mathcal{O}_{0}\right)$, i.e.,

$$
\begin{aligned}
& \mathbb{P}\left(\left|h_{i d}\right|^{2}<\rho^{r-1}\right) \dot{\geq} \mathbb{P}\left(\mathcal{O}_{i} \mid \mathcal{O}_{0}, \ldots, \mathcal{O}_{i-1}\right) \geq \mathbb{P}\left(\mathcal{O}_{i} \mid \mathcal{O}_{0}\right) \\
& \rho^{r-1} \dot{\geq} \mathbb{P}\left(\mathcal{O}_{i} \mid \mathcal{O}_{0}, \ldots, \mathcal{O}_{i-1}\right) \dot{\geq} \rho^{r-1} \text {. }
\end{aligned}
$$

Hence, $d_{i}^{\prime}(r)=(1-r)^{+}$for $1<i \leq n$. Substituting the conditional DMTs into Equation (5) completes the proof.

Note that the above selection procedures are designed to be DMT-optimal and to simplify the analysis. Selecting the mode $i^{*}=\arg \max _{i \in\{0, \ldots, n\}} I_{i}$ gives the same DMT.

2) Opportunistic Amplify and Forward Orthogonal Relaying: If the direct link is in outage, the selected relay amplifies its received signal during the first half-interval and forwards it to the destination in the second half-interval. The instantaneous mutual information of the non-relay mode is given by $I_{0}=\log \left(1+\left|h_{s d}\right|^{2} \rho\right)$. The end-to-end instantaneous mutual information for the relay-assisted modes under orthogonal AF is given by [20], [21]

$I_{i}=\frac{1}{2} \log \left(1+\left|h_{s d}\right|^{2} \rho+f\left(\left|h_{s i}\right|^{2} \rho,\left|h_{i d}\right|^{2} \rho\right)\right), \quad i=1, \ldots, n$, where $f(x, y)=\frac{x y}{x+y+1}$. At high SNR, $f\left(\left|h_{s i}\right|^{2} \rho,\left|h_{i d}\right|^{2} \rho\right)$ can be approximated to $\frac{\left|h_{s i}\right|^{2}\left|h_{i d}\right|^{2} \rho}{\left|h_{s i}\right|^{2}|| h_{i d} \mid}$ and relay $i^{*}$ is selected such that $i^{*}=\arg \max _{i} \frac{\left|h_{s i}\right|^{2}\left|h_{i d}\right|^{2}}{\left|h_{s i}\right|^{2}+\left|h_{i d}\right|^{2}}$.

Theorem 3: The DMT of the orthogonal opportunistic AF parallel relay channel with direct link between the source and the destination is given by

$$
d(r)=(1-r)^{+}+n(1-2 r)^{+} .
$$

Proof: The overall DMT in terms of individual conditional DMTs is given by Equation (5). To calculate the right hand side, we start with the non-relay mode (Mode 0) whose DMT is $d_{0}(r)=(1-r)^{+}$. At high SNR, the conditional outage of Mode 1 is

$$
\mathbb{P}\left(\mathcal{O}_{1} \mid \mathcal{O}_{0}\right) \doteq \mathbb{P}\left(\left\{\left|h_{s d}\right|^{2}+\frac{\left|h_{s 1}\right|^{2}\left|h_{1 d}\right|^{2}}{\left|h_{s 1}\right|^{2}+\left|h_{1 d}\right|^{2}}<\frac{\rho^{2 r}-1}{\rho}\right\} \mid\right.
$$



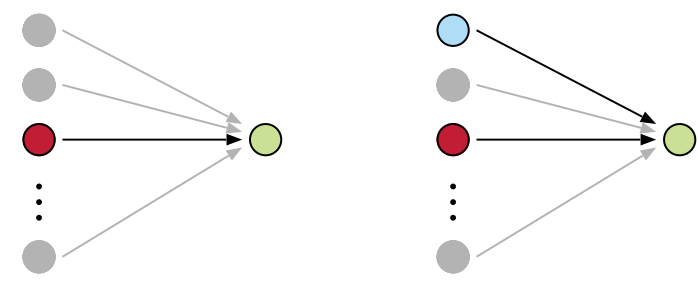

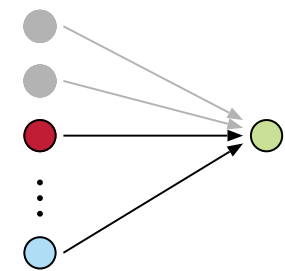

Fig. 3. The non-orthogonal genie upper bound access modes.

$$
\left.\left\{\left|h_{s d}\right|^{2}<\frac{\rho^{r}-1}{\rho}\right\}\right)
$$

In order to calculate the conditional outage probability distribution, we first calculate the conditional density function of $Z=\left|h_{s d}\right|^{2}+V$ where $V=\frac{\left|h_{s 1}\right|^{2}\left|h_{1 d}\right|^{2}}{\left|h_{s 1}\right|^{2}+\left|h_{1 d}\right|^{2}}$. The term $\frac{\left|h_{s 1}\right|^{2}\left|h_{1 d}\right|^{2}}{\left|h_{s 1}\right|^{2}+\left|h_{1 d}\right|^{2}}$ represents half the harmonic mean of two independent exponential random variables. Using a result of [22], the harmonic mean of two exponential random variables with parameters $\lambda$ can be approximated by an exponential random variable with parameter $\lambda+\lambda=2 \lambda$.

Using results from the Appendix and defining $\alpha=$ $g_{1}(r, \rho)=\frac{\rho^{r}-1}{\rho}$ and $g_{2}(r, \rho)=\frac{\rho^{2 r}-1}{\rho}$, the conditional outage probability is

$$
\begin{aligned}
\mathbb{P}\left(\mathcal{O}_{1} \mid \mathcal{O}_{0}\right)= & \int_{0}^{g_{2}(r, \rho)} f_{Z \mid B}(z) d z \\
\doteq & 2 \int_{0}^{g_{1}(r, \rho)} \frac{e^{-2 z}\left(e^{z}-1\right)}{1-e^{-g_{1}(r, \rho)}} d z \\
& \quad+2 \int_{g_{1}(r, \rho)}^{g_{2}(r, \rho)} \frac{e^{-2 z}\left(e^{g_{1}(r, \rho)}-1\right)}{1-e^{-g_{1}(r, \rho)}} d z \\
= & \frac{e^{-2 \rho^{2 r-1}}-e^{-\rho^{r-1}}-e^{-2 \rho^{2 r-1}+\rho^{r-1}}+1}{1-e^{-\rho^{r-1}}} \\
\doteq & \rho^{2 r-1} .
\end{aligned}
$$

From Equations (14) and (8), $d_{1}^{\prime}(r)=(1-2 r)^{+}$.

For Mode 2, the conditional outage can be shown to be

$$
\mathbb{P}\left(\mathcal{O}_{2} \mid \mathcal{O}_{1}, \mathcal{O}_{0}\right) \geq \mathbb{P}\left(\mathcal{O}_{2} \mid \mathcal{O}_{0}\right) \doteq \rho^{2 r-1},
$$

where (15) follows the same proof as $\mathbb{P}\left(\mathcal{O}_{1} \mid \mathcal{O}_{0}\right)$. Also one can show

$$
\begin{aligned}
& \mathbb{P}\left(\mathcal{O}_{2} \mid \mathcal{O}_{1}, \mathcal{O}_{0}\right) \\
& \doteq \mathbb{P}\left(\left\{\left|h_{s d}\right|^{2}+f\left(\left|h_{s 2}\right|^{2},\left|h_{2 d}\right|^{2}\right)<\rho^{2 r-1}\right\} \mid \mathcal{O}_{1}, \mathcal{O}_{0}\right) \\
& \dot{\leq} \mathbb{P}\left(\left\{f\left(\left|h_{s 2}\right|^{2},\left|h_{2 d}\right|^{2}\right)<\rho^{2 r-1}\right\} \mid \mathcal{O}_{1}, \mathcal{O}_{0}\right) \\
& \dot{\leq} \mathbb{P}\left(f\left(\left|h_{s 2}\right|^{2},\left|h_{2 d}\right|^{2}\right)<\rho^{2 r-1}\right) \doteq \rho^{2 r-1} .
\end{aligned}
$$

Hence, $\mathbb{P}\left(\mathcal{O}_{2} \mid \mathcal{O}_{1}, \mathcal{O}_{0}\right) \doteq \rho^{2 r-1}$ and $d_{2}^{\prime}(r)=(1-2 r)^{+}$. Following similar steps, one can show that $d_{i}^{\prime}(r)=(1-2 r)^{+}$for $i=3, \ldots, n$. Substituting conditional DMTs into Equation (5) completes the proof.

3) Opportunistic Decode and Forward Orthogonal Relaying: If the direct link is not capable of supporting the transmission rate, the transmission interval is divided into two intervals. In the first transmission interval, the source transmits. If some relays decode the source message, then a relay will forward the decoded message to the destination in the second half of the transmission interval and the source remains silent. If neither the non-relayed mode nor any relayed mode is capable of supporting the desired rate, the system is in outage.

Theorem 4: The DMT of the orthogonal opportunistic DF parallel relay channel with direct link between the source and the destination is given by

$$
d(r)=(1-r)^{+}+n(1-2 r)^{+}
$$

Proof: The total DMT as a function of conditional DMTs is given by Equation (5) and $d_{0}(r)=(1-r)^{+}$. The conditional outage probability of the first relay-assisted mode is given by

$$
\begin{aligned}
\mathbb{P}\left(\mathcal{O}_{1} \mid \mathcal{O}_{0}\right)=\mathbb{P}( & \left\{\frac{1}{2} \log \left(1+U_{1} \rho\right)<r \log \rho\right\} \mid \\
& \left.\left\{\log \left(1+\left|h_{s d}\right|^{2} \rho\right)<r \log \rho\right\}\right),
\end{aligned}
$$

where the random variable $U_{1}$ is defined via

$$
\begin{gathered}
U_{i}= \begin{cases}\left|h_{s d}\right|^{2} & \left|h_{s i}\right|^{2}<\frac{\rho^{2 r}-1}{\rho} \\
\left|h_{s d}\right|^{2}+\left|h_{i d}\right|^{2} & \left|h_{s i}\right|^{2} \geq \frac{\rho^{2 r}-1}{\rho} .\end{cases} \\
\mathbb{P}\left(\mathcal{O}_{1} \mid \mathcal{O}_{0}\right)=\mathbb{P}\left(\left\{\left|h_{s d}\right|^{2}<\frac{\rho^{2 r}-1}{\rho}\right\} \mid\left\{\left|h_{s d}\right|^{2}<\frac{\rho^{r}-1}{\rho}\right\}\right) \\
\times \mathbb{P}\left(\left|h_{s 1}\right|^{2}<\frac{\rho^{2 r}-1}{\rho}\right) \\
+\mathbb{P}\left(\left\{\left|h_{s d}\right|^{2}+\left|h_{1 d}\right|^{2}<\frac{\rho^{2 r}-1}{\rho}\right\} \mid\left\{\left|h_{s d}\right|^{2}<\frac{\rho^{r}-1}{\rho}\right\}\right) \\
\times \mathbb{P}\left(\left|h_{s 1}\right|^{2} \geq \frac{\rho^{2 r}-1}{\rho}\right) .
\end{gathered}
$$

One can show that $\frac{\rho^{2 r}-1}{\rho}>\frac{\rho^{r}-1}{\rho}$, therefore

$$
\mathbb{P}\left(\left\{\left|h_{s d}\right|^{2}<\frac{\rho^{2 r}-1}{\rho}\right\} \mid\left\{\left|h_{s d}\right|^{2}<\frac{\rho^{r}-1}{\rho}\right\}\right) \doteq 1 .
$$

Using results from the Appendix and defining $g_{1}(r, \rho) \triangleq \frac{\rho^{r}-1}{\rho}$ and $g_{2}(r, \rho) \triangleq \frac{\rho^{2 r}-1}{\rho}$

$$
\begin{aligned}
& \mathbb{P}\left(\left\{\left|h_{s d}\right|^{2}+\left|h_{1 d}\right|^{2}<g_{2}(r, \rho)\right\} \mid\left\{\left|h_{s d}\right|^{2}<g_{1}(r, \rho)\right\}\right) \\
& =\int_{0}^{g_{1}(r, \rho)} \frac{z e^{-z}}{1-e^{-g_{1}(r, \rho)}} d z+\int_{g_{1}(r, \rho)}^{g_{2}(r, \rho)} \frac{g_{1}(r, \rho) e^{-z}}{1-e^{-g_{1}(r, \rho)}} d z \\
& \doteq \frac{1-e^{-\rho^{r-1}}-\rho^{r-1} e^{-\rho^{2 r-1}}}{1-e^{-\rho^{r-1}}} \doteq \rho^{2 r-1}
\end{aligned}
$$


Substituting (22) and (23) into (21), the conditional probability of outage is given by

$$
\mathbb{P}\left(\mathcal{O}_{1} \mid \mathcal{O}_{0}\right) \doteq \rho^{2 r-1}+\rho^{2 r-1}\left(1-\rho^{2 r-1}\right) \doteq \rho^{2 r-1} .
$$

Hence, the conditional DMT for the first relay-assisted access mode is $d_{1}^{\prime}(r)=(1-2 r)^{+}$. Using the same argument as the orthogonal $\mathrm{AF}$, it follows that $\mathbb{P}\left(\mathcal{O}_{i} \mid \mathcal{O}_{0}, \ldots, \mathcal{O}_{i-1}\right) \doteq \rho^{2 r-1}$ and hence $d_{i}^{\prime}(r)=(1-2 r)^{+}$for $i=2, \ldots, n$. Substituting conditional DMTs into Equation (5) completes the proof.

4) Heterogeneous Network with $A F$ and DF Orthogonal Relays: In this scenario both $\mathrm{AF}$ and $\mathrm{DF}$ relays are present in the network. At high SNR, the selected relay $i^{*}$ is such that $i^{*}=\arg \max _{i}\left\{\gamma_{i \in D}^{D F}, \gamma_{i \in A}^{A F}\right\}$, where $D$ and $A$ represent the DF and AF relays set, respectively, and $\gamma_{i}$ is the SNR at the destination when Relay $i$ is active.

Theorem 5: The DMT of the orthogonal opportunistic relay selection with $N$ AF relay and $M$ DF relays channel and a direct link between the source and the destination is given by

$$
d(r)=(1-r)^{+}+(N+M)(1-2 r)^{+} .
$$

Proof: The total DMT as a function of conditional DMTs is given by Equation (5). As mentioned earlier for the nonrelay mode (Mode 0) DMT is $d_{0}(r)=(1-r)^{+}$.

We know there is at least one AF and one DF relay in this network. For the purposes of exposition, we assign Mode 1 to an AF relay and Mode 2 to a DF relay. For Mode 1, at high SNR, the analysis is identical to Mode 1 analysis in Section IV-A2. From Equation (14), the conditional outage $\mathbb{P}\left(\mathcal{O}_{1} \mid \mathcal{O}_{0}\right) \doteq \rho^{2 r-1}$, hence for Mode 1 , the conditional DMT is $d_{1}^{\prime}(r)=(1-2 r)^{+}$.

For Mode 2, we first bound the conditional outage from below:

$$
\mathbb{P}\left(\mathcal{O}_{2} \mid \mathcal{O}_{1}, \mathcal{O}_{0}\right) \geq \mathbb{P}\left(\mathcal{O}_{2} \mid \mathcal{O}_{0}\right)
$$

The calculation of $\mathbb{P}\left(\mathcal{O}_{2} \mid \mathcal{O}_{0}\right)$ is similar to Equation (24) and can be shown to be

$$
\begin{aligned}
\mathbb{P}\left(\mathcal{O}_{2} \mid \mathcal{O}_{0}\right) & =\mathbb{P}\left(\left\{\frac{1}{2} \log \left(1+U_{2} \rho\right)<r \log \rho\right\} \mid\right. \\
& \left.\quad\left\{\log \left(1+\left|h_{s d}\right|^{2} \rho\right)<r \log \rho\right\}\right) \\
& \doteq \rho^{2 r-1} .
\end{aligned}
$$

Now, we bound $\mathbb{P}\left(\mathcal{O}_{2} \mid \mathcal{O}_{1}, \mathcal{O}_{0}\right)$ from above, using the same technique used in Equation (10)

$$
\mathbb{P}\left(\mathcal{O}_{2} \mid \mathcal{O}_{1}, \mathcal{O}_{0}\right) \dot{\leq} \mathbb{P}\left(U_{2}<\rho^{2 r-1}\right) \doteq \rho^{2 r-1}
$$

Thus, using a sandwich argument, we have $\mathbb{P}\left(\mathcal{O}_{2} \mid \mathcal{O}_{1}, \mathcal{O}_{0}\right) \doteq$ $\rho^{2 r-1}$, indicating the conditional DMT of Mode 2 is $d_{2}^{\prime}(r)=$ $(1-2 r)^{+}$. Following the same steps, one can show that $d_{i}^{\prime}(r)=(1-2 r)^{+}$for $i=3, \ldots, N+M$. Substituting the conditional DMTs into Equation (5) completes the proof.

\section{B. Non-Orthogonal Relaying}

In this scenario the source transmits throughout the transmission interval. The relays listens for a portion of the transmission interval and then, depending on the channel conditions, one of the relays may transmit simultaneously with the source for a portion of the transmission interval. The destination receives the source signal, potentially superimposed with the relay signal. Since in non-orthogonal modes the end-to-end mutual information always improves with a relay, there is no need to consider a non-relay access mode for the purposes of DMT calculation. Thus in the analysis of non-orthogonal protocols, the system has $n$ access modes as opposed to $n+1$ in the case of orthogonal relaying.

1) Upper Bound: For the calculation of the upper bound we assume a genie provides the relays with a noise-free and error-free version of the source message. We also assume full cooperation between the source and the relays. The relay that maximizes the instantaneous end-to-end mutual information is selected to transmit simultaneously with the source. Each of the $n$ relays has an independent link to the destination and they all share the same source-destination link. Subject to the genie information, the distinction between relay types (AF, $\mathrm{DF}, \mathrm{CF}$ ) goes away and therefore the upper bound is equally valid for any set of $n$ non-orthogonal relays.

Theorem 6: An upper bound for the opportunistic parallel relay channel with $n$ non-orthogonal relays is

$$
d(r)=(n+1)(1-r)^{+} .
$$

Proof: The genie-aided opportunistic parallel relay channel is equivalent to a MISO system with $n+1$ transmit antennas and one receive antenna. The performance of the opportunistic genie-aided relay selection is therefore upper bounded by a $(n+1) \times 1$ MISO system with antenna selection that chooses for each codeword transmission two transmit antennas. The $(n+1) \times 1$ antenna selection allows configurations that do not have a counterpart in the opportunistic modes in our channel, therefore in addition to the genie we have allowed a second relaxation of conditions for the computation of the upper bound in this theorem.

The DMT of a $M \times N$ MIMO link with $L_{t}<M$ selected transmit antennas and $L_{r}<N$ selected receive antennas is upper bounded by a piecewise linear function obtained by connecting the following $K+2$ points [23]

$$
\left\{\left(n,\left(M_{r}-n\right)\left(M_{t}-n\right)\right)\right\}_{n=0}^{K},\left(\min \left(L_{r}, L_{t}\right), 0\right),
$$

where

$$
\begin{aligned}
K= & \arg \min _{k \in \mathbb{Z}} \frac{\left(M_{r}-k\right)\left(M_{t}-k\right)}{\min \left(L_{r}, L_{t}\right)-k}, \\
& \text { subject to } 0 \leq k \leq \min \left(L_{r}, L_{t}\right)-1
\end{aligned}
$$

Using this result, a $(n+1) \times 1$ MISO system with two selected transmit antennas has a DMT that is upper bounded by

$$
d(r)=(n+1)(1-r)^{+} .
$$

This represents our genie-aided upper bound.

2) Non-Orthogonal Amplify and Forward: For half the transmission interval, the received signal at the destination and at Relay $i$ are given by

$$
y_{1 d}=\sqrt{\rho} h_{s d} x_{1 s}+n_{1 d}, \quad y_{1 i}=\sqrt{\rho} h_{s i} x_{1 s}+n_{1 i} .
$$

The variables $x, y, n$ represent source transmitted signal, received signal and the noise, respectively. The variables have two subscripts indicating the appropriate half-interval and 
node identity, respectively. At the second half of the transmission interval the relay normalizes the received signal and retransmits it. The destination received signal in the second half is

$$
y_{2 d}=\sqrt{\rho} h_{s d} x_{2 s}+\frac{\sqrt{\rho} h_{i d}}{\sqrt{\rho\left|h_{s i}\right|^{2}+1}} y_{1 i}+n_{2 d} .
$$

At the destination $Y=\mathbf{H}_{i} X+N$ where $X=\left[\begin{array}{ll}x_{1 s} & x_{2 s}\end{array}\right]^{t}$ is the transmitted signals vector, $N$ is the noise vector, and $\mathbf{H}_{i}$ is the effective channel gain matrix [24].

$$
\mathbf{H}_{i}=\left[\begin{array}{cc}
\sqrt{\rho} h_{s d} & 0 \\
\frac{\rho h_{i d} h_{s i}}{\sqrt{\rho\left|h_{s i}\right|^{2}+1} \sqrt{\frac{\rho\left|h_{i d}\right|^{2}}{\rho\left|h_{s i}\right|^{2}+1}+1}} & \frac{\sqrt{\rho} h_{s d}}{\sqrt{\frac{\rho\left|h_{i d}\right|^{2}}{\rho\left|h_{s i}\right|^{2}+1}+1}}
\end{array}\right] .
$$

The instantaneous mutual information is given by

$$
\begin{aligned}
I_{i}= & \frac{1}{2} \log \left|\mathbf{I}+\mathbf{H}_{i} \mathbf{H}_{i}^{*}\right| \\
= & \frac{1}{2} \log \left(1+\left|h_{s d}\right|^{2} \rho+\frac{\left|h_{i d}\right|^{2}\left|h_{s i}\right|^{2} \rho^{2}}{\left|h_{i d}\right|^{2} \rho+\left|h_{s i}\right|^{2} \rho+1}\right. \\
& \left.\quad+\frac{\left|h_{s d}\right|^{2}\left(\left|h_{s i}\right|^{2} \rho+1\right) \rho}{\left|h_{i d}\right|^{2} \rho+\left|h_{s i}\right|^{2} \rho+1}+\frac{\left|h_{s d}\right|^{4}\left(\left|h_{s i}\right|^{2} \rho+1\right) \rho^{2}}{\left|h_{i d}\right|^{2} \rho+\left|h_{s i}\right|^{2} \rho+1}\right) .
\end{aligned}
$$

Relay $i^{*}$ is selected to maximize the mutual information, which at high SNR is:

$$
i^{*}=\arg \max _{i} I_{i}=\arg \max _{i}\left\{\frac{\left|h_{s i}\right|^{2}}{\left|h_{i d}\right|^{2}}\right\} .
$$

Theorem 7: The maximum DMT for the $n$ parallel relay channel with opportunistic NAF relay selection is

$$
d(r)=(1-r)^{+}+n(1-2 r)^{+} .
$$

Proof: The DMT of a system that switches between $n$ dependent access modes is given by

$$
d(r)=-\lim _{\rho \rightarrow \infty} \frac{\log \mathbb{P}\left(\mathcal{O}_{1}, \ldots, \mathcal{O}_{n}\right)}{\log \rho} .
$$

In a manner similar to [25], the probability of outage $\mathbb{P}\left(\mathcal{O}_{1}, \ldots, \mathcal{O}_{n}\right)$ can be expressed as follows

$$
\begin{gathered}
\mathbb{P}\left(\mathcal{O}_{1}, \ldots, \mathcal{O}_{n}\right) \doteq \rho^{-d_{o}(r)} \\
d_{o}(r)=\inf _{\left(v_{1}, u^{(1)}, v_{2}^{(1)}, \ldots, u_{1}^{(n)}, v_{2}^{(n)}\right) \in O} v_{1}+\sum_{i=1}^{n}\left(u^{(i)}+v_{2}^{(i)}\right) .
\end{gathered}
$$

The random variables $v_{1}, u^{(i)}$ and $v_{2}^{(i)}$ represent the exponential order of $1 /\left|h_{s d}\right|^{2}, 1 /\left|h_{s i}\right|^{2}$ and $1 /\left|h_{i d}\right|^{2}$, respectively. Each of these random variables has a probability density function $p(x)$ that is asymptotically equal to $\rho^{-x}$ for $x \geq 0$ and 0 otherwise [25]. The set $O$ represents the outage event for the network. The network is considered in outage when no access mode is viable, i.e., $O=\mathcal{O}_{1}^{+} \cap \ldots \cap \mathcal{O}_{n}^{+}$. For NAF, the outage region for Mode $i$ is defined by [25]

$$
\begin{aligned}
& \mathcal{O}_{i}^{+}=\left\{\left(v_{1}, v_{2}^{(i)}, u^{(i)}\right) \in R^{3+} \mid\left(l-2 m^{(i)}\right)\left(1-v_{1}\right)^{+}\right. \\
& \left.\left.+m^{(i)} \max \left\{2\left(1-v_{1}\right), 1-\left(v_{2}^{(i)}+u^{(i)}\right)\right\}\right)^{+}<r l\right\},
\end{aligned}
$$

where $m^{(i)}$ is rank of the amplification matrix and $l$ is the block length. The solution to the optimization problem in Equations (37) and (38) is facilitated by the knowledge that $d_{o}(r)$ is maximized when $m^{(i)}$ is maximum, i.e, $m^{(i)}=l / 2$, thus leading to the result.
3) Dynamic Decode and Forward: In this scenario the selected relay listens to the source until it has enough information to decode the message. From that point on, the relay uses the remainder of the transmission interval to send the decoded information to the destination. The selection criterion is, once again, according to the maximum end-to-end mutual information.

Theorem 8: The maximum DMT for the $n$ parallel relay channel with opportunistic DDF relay selection is

$$
d_{D D F}(r)= \begin{cases}(n+1)(1-r) & \frac{1}{n+1} \geq r \geq 0 \\ (n+1)-n \frac{r}{1-r} & \frac{1}{2} \geq r>\frac{1}{n+1} \\ \frac{1-r}{r} & r \geq \frac{1}{2} .\end{cases}
$$

Proof: Following Equations (35), (36), and (37), for the relay channel characterized by Relay $i$, the outage region under DDF can be shown to be [25]

$$
\begin{aligned}
\mathcal{O}_{i}^{+}= & \left\{\left(v_{1}, v_{2}^{(i)}, u^{(i)}\right) \in R^{3+} \mid t_{i}\left(1-v_{1}\right)^{+}\right. \\
& \left.+\left(1-t_{i}\right)\left(1-\min \left(v_{1}, v_{2}^{(i)}\right)\right)^{+}<r\right\},
\end{aligned}
$$

where $t_{i}$ is the listening-time ratio of the relay $i$, with $r \leq t_{i} \leq$ 1. In the following we outline the solution of Equations (37) and (40) for a two-relay channel. The generalization to $n$ users follows the same lines.

Our strategy for solving the optimization problem is to partition the optimization space into eight regions, solve the optimization problem over each region as a function of $t_{1}$ and $t_{2}$, maximize over $t_{1}$ and $t_{2}$ and then find the minimum of the eight solutions. The eight regions correspond to the Cartesian product of whether each of the three positive variables $v_{1}^{(1)}, v_{1}^{(2)}, v_{2}$ is greater than or less than 1 . Following the calculations, which are omitted due to their length, the DMT for DDF is established.

4) Compress and Forward: In this scenario, following [26], the selected relay uses Wyner-Ziv compression and the destination uses the received signal from the source as side information to decode the signal. The compression rate ensures that the compressed signal is decoded at the destination errorfree. Yuksel and Erkip [26] show that the one-relay compressforward achieves the DMT $d(r)=2(1-r)^{+}$, which coincides with the MISO upper bound.

The relay $i^{*}=\arg \max _{i} I_{i}$ is selected which at high-SNR, using results from [26], is:

$$
i^{*}=\arg \max _{i} \frac{\left(\left|h_{s i}\right|^{2}+\left|h_{s d}\right|^{2}\right)\left(\left|h_{i d}\right|^{2}+\left|h_{s d}\right|^{2}\right)}{\left(\left|h_{s i}\right|^{2}+\left|h_{s d}\right|^{2}\right)+\left(\left|h_{i d}\right|^{2}+\left|h_{s d}\right|^{2}\right)} .
$$

Following [26], the DMT for the $n$ parallel relay channel with opportunistic CF relay selection can be shown to be

$$
\begin{aligned}
d(r) & =\max _{t} \min \left(d_{M A C}(r, t), d_{B C}(r, t)\right), \\
d_{M A C} & =-\lim _{\rho \rightarrow \infty} \frac{\log \min _{p\left(x_{s}, x_{r} \mid q\right)} \mathbb{P}\left(I_{M A C}<r \log \rho\right)}{\log \rho}, \\
d_{B C} & =-\lim _{\rho \rightarrow \infty} \frac{\log \min _{p\left(x_{s}, x_{r} \mid q\right)} \mathbb{P}\left(I_{B C}<r \log \rho\right)}{\log \rho},
\end{aligned}
$$

where $t$ is the time ratios vector, $t=\left[t_{1}, \ldots, t_{n}\right], q$ represents the state of the relay (listening vs. transmitting), $p\left(x_{s}, x_{r} \mid q\right)$ is the probability density according to which the random 
codebooks are generated for the source and the relay, and $I_{B C}$ and $I_{M A C}$ represent the total mutual information across the source and the destination cutsets, respectively. We have:

$$
\begin{aligned}
I_{M A C} \leq(1 & \left.-t_{i^{*}}\right) \log \left(1+\left|h_{s d}\right|^{2} \rho\right) \\
& +t_{i^{*}} \log \left(1+\left(\left|h_{s d}\right|^{2}+\left|h_{i^{*} d}\right|^{2}\right) \rho\right) .
\end{aligned}
$$

We can show that $\mathbb{P}\left(I_{M A C}<r \log \rho\right) \doteq \rho^{-d_{M A C}(r)}$ where

$$
d_{M A C}(r)=\inf _{\left(v_{1}, v_{2}^{(1)}, \ldots, v_{2}^{(n)}\right) \in O} v_{1}+\sum_{i=1}^{n} v_{2}^{(i)},
$$

and the outage event $\mathrm{O}_{i}^{+}$is defined as

$$
\begin{aligned}
O_{i}^{+}=\left\{\left(v_{1}, v_{2}^{(i)}\right) \in\right. & R^{2+} \mid\left(1-t_{i}\right)\left(1-v_{1}\right)^{+}+ \\
& \left.t_{i}\left(1-\min \left(v_{1}, v_{2}^{(i)}\right)\right)^{+} \leq r\right\} .
\end{aligned}
$$

Solving the optimization problem in (42), the DMT for the destination cutset is (44 on the next page) where $\Gamma=\left\{t_{i}\right.$ : $\left.t_{i}<1-r\right\}$, and the third line in Eq. (44) represents multiple expressions, one for each $t_{i}$ that satisfies the corresponding condition. The details of the optimization are omitted for brevity.

Similarly, the source cutset DMT can be obtained by replacing $t_{i}$ with $1-t_{i}$. Maximizing over $t$, the minimum of the two DMT cutsets gives the DMT of the system.

Thus, the DMT for arbitrary number of relays is a composite expression which does not reduce to a more compact form as long as the number of relays remains a variable. However, for any fixed number of relays the results are more streamlined. For example, for selection among three $\mathrm{CF}$ relays:

$$
d_{C F}(r)= \begin{cases}4-6 r & r<\frac{1}{3} \\ 3(1-r) & \frac{1}{3} \leq r \leq 1\end{cases}
$$

5) Mixture of NAF and DDF Relays: Now consider a scenario consisting of $N$ NAF relays and $M$ DDF relays. If the selected relay is an NAF relay, the relay will listen for half the transmission interval and transmits simultaneously with the source in the second half of the transmission interval. If the selected relay is a DDF relay, the relay listens to the source until it has enough information to decode the message, then transmits during the remainder of the transmission interval.

Theorem 9: The DMT for an opportunistic multiple relay channel with $N$ NAF relays and $M$ DDF relays is given by

$$
d(r)= \begin{cases}(M+1)(1-r)+N(1-2 r) & \frac{1}{M+1} \geq r \geq 0 \\ (M+1)-\frac{M r}{1-r}+N(1-2 r) & \frac{1}{2} \geq r \geq \frac{1}{M+1} \\ \frac{1-r}{r} & 1 \geq r \geq \frac{1}{2} .\end{cases}
$$

Proof: The DMT of a system that switches between $N+$ $M$ dependent access modes is given by

$$
d(r)=-\lim _{\rho \rightarrow \infty} \frac{\log \mathbb{P}\left(\mathcal{O}_{1}, \ldots, \mathcal{O}_{N+M}\right)}{\log \rho} .
$$

In a manner similar to [25], the probability of outage $\mathbb{P}\left(\mathcal{O}_{1}, \ldots, \mathcal{O}_{n}\right)$ can be expressed as follows

$$
\mathbb{P}\left(\mathcal{O}_{1}, \ldots, \mathcal{O}_{n}\right) \doteq \rho^{-d_{o}(r)},
$$

where

$$
d_{o}(r)=\inf _{\left(v_{1}, u^{(i)}, v_{2}^{(i)}\right) \in O} v_{1}+\sum_{i=1}^{N+M}\left(u^{(i)}+v_{2}^{(i)}\right),
$$

where $O=\mathcal{O}_{1}^{+} \cap \ldots \cap \mathcal{O}_{N+M}^{+}$.

For the purposes of exposition, the NAF access modes are indexed as Mode $i$ where $i=1, \ldots, N$. The outage region can be shown to be [25]

$$
\begin{aligned}
\mathcal{O}_{i}^{+}= & \left\{\left(v_{1}, v_{2}^{(i)}, u^{(i)}\right) \in R^{3+} \mid\left(l-2 m^{(i)}\right)\left(1-v_{1}\right)^{+}\right. \\
& \left.\left.+m^{(i)} \max \left\{2\left(1-v_{1}\right), 1-\left(v_{2}^{(i)}+u^{(i)}\right)\right\}\right)^{+}<r l\right\},
\end{aligned}
$$

where $m^{(i)}$ is rank of the amplification matrix and $l$ is the block length.

The DDF access modes are indexed as Mode $\mathrm{i}$ where $i=$ $N+1, \ldots, N+M$. The outage region can be shown to be [25]

$$
\begin{aligned}
\mathcal{O}_{i}^{+}=\{ & \left(v_{1}, v_{2}^{(i)}, u^{(i)}\right) \in R^{3+} \mid t_{i}\left(1-v_{1}\right)^{+} \\
& \left.+\left(1-t_{i}\right)\left(1-\min \left(v_{1}, v_{2}^{(i)}\right)\right)^{+}<r\right\},
\end{aligned}
$$

where $t_{i}$ is the listening-time ratio of the relay $i$, with $r \leq$ $t_{i} \leq 1$.

The DMT is found by solving the optimization problem in Equation (49) over the union of the regions defined in Equations (50) and (51). The details of the optimization are omitted for brevity.

6) Mixture of $N A F$ and CF Relays: In this scenario we have $N$ NAF relays and $M$ CF relays. The NAF relays work as mentioned in the previous subsection. For CF relays, we again follow [26] where the CF relays use Wyner-Ziv compression and the destination uses the received signal from the source as side information to decode the signal. As mentioned earlier, a single-relay CF channel achieves the DMT $d(r)=2(1-r)^{+}$.

The relay $i^{*}=\arg \max _{i} I_{i}$ is selected. At high-SNR, using results from [26] and [24], the selected user $i^{*}$ is

$$
\begin{aligned}
i^{*}= & \arg \max _{i}\left\{\left\{\frac{\left|h_{s i}\right|^{2}}{\left|h_{i d}\right|^{2}}\right\}_{i=1, \ldots, N},\right. \\
& \left.\left\{\frac{\left(\left|h_{s i}\right|^{2}+\left|h_{s d}\right|^{2}\right)\left(\left|h_{i d}\right|^{2}+\left|h_{s d}\right|^{2}\right)}{\left(\left|h_{s i}\right|^{2}+\left|h_{s d}\right|^{2}\right)+\left(\left|h_{i d}\right|^{2}+\left|h_{s d}\right|^{2}\right)}\right\}_{i=N+1, \ldots, N+M}\right\},
\end{aligned}
$$

where without loss of generality we have indexed the relays so that the first $N$ relays are NAF and the following $M$ relays are $\mathrm{CF}$.

We provide a sketch of the technique used to arrive at the DMT. Following [26], the DMT for the multiple relay channel with opportunistic relay selection can be shown to be

$$
\begin{aligned}
d(r) & =\max _{t} \min \left(d_{C F}(r, t), d_{B C}(r, t)\right), \\
d_{M A C} & =-\lim _{\rho \rightarrow \infty} \frac{\log \mathbb{P}\left(I_{C F-M A C}<r \log \rho, I_{N A F}<r \log \rho\right)}{\log \rho}, \\
d_{B C} & =-\lim _{\rho \rightarrow \infty} \frac{\log \mathbb{P}\left(I_{C F-B C}<r \log \rho, I_{N A F}<r \log \rho\right)}{\log \rho},
\end{aligned}
$$

where $t$ is the time ratios vector, $t=\left[t_{N+1}, \ldots, t_{N+M}\right]$, for the CF relays, and $I_{B C}$ and $I_{M A C}$ represent the total mutual information across the source and the destination 


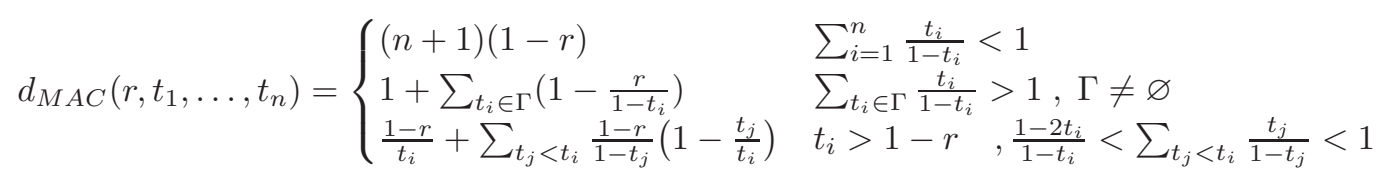

cutsets considering one CF relay active, respectively. It can be shown that

$$
\begin{aligned}
I_{C F M A C} \leq(1- & \left.t_{i^{*}}\right) \log \left(1+\left|h_{s d}\right|^{2} \rho\right) \\
& +t_{i^{*}} \log \left(1+\left(\left|h_{s d}\right|^{2}+\left|h_{i^{*} d}\right|^{2}\right) \rho\right) .
\end{aligned}
$$

The outage probability can be expressed as

$$
\mathbb{P}\left(I_{C F M A C}<r \log \rho, I_{N A F}<r \log \rho\right) \doteq \rho^{-d(r)},
$$

where

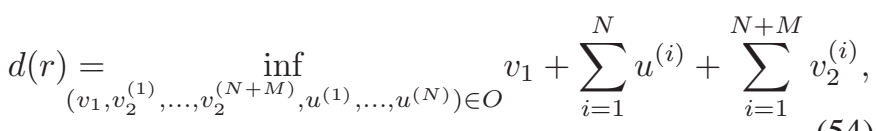

For $i=1, \ldots, N$, the NAF relay assisted modes, the outage event $O_{i}^{+}$is defined as given by Equation (50). For $i=\{N+$ $1, \ldots, N+M\}$, the $\mathrm{CF}$ relay assisted modes, the outage event $\mathrm{O}_{i}^{+}$is defined as

$$
\begin{aligned}
O_{i}^{+}=\left\{\left(v_{1}, v_{2}^{(i)}\right) \in\right. & R^{2+} \mid\left(1-t_{i}\right)\left(1-v_{1}\right)^{+} \\
& \left.+t_{i}\left(1-\min \left(v_{1}, v_{2}^{(i)}\right)\right)^{+} \leq r\right\} .
\end{aligned}
$$

We provide the solution for the optimization problem for the case of $2 \mathrm{CF}$ relays and $N$ NAF. the generalization to the $M$ $\mathrm{CF}$ relays follows along the same lines.

$$
d(r)= \begin{cases}(3-4 r)+N(1-2 r) & \frac{3}{7} \geq r \geq 0 \\ \frac{9}{4}(1-r)+N(1-2 r) & \frac{1}{2} \geq r \geq \frac{3}{7} \\ \frac{9}{4}(1-r) & 1 \geq r \geq \frac{1}{2}\end{cases}
$$

Figure 4 compares the opportunistic DMT of a system consists of one source, one destination and two relays with a direct link between the source and the destination. Figure 5 shows the DMT for the case of three relays, where various combinations of three available relays are analyzed. Figure 6 considers a configuration with four relays, where the relays can be all ODF, all OAF, all NAF, all DDF, a mix of 2 OAF and $2 \mathrm{ODF}$, a mix of $2 \mathrm{NAF}$ and $2 \mathrm{DDF}$, or a mix of 2 $\mathrm{NAF}$ and $2 \mathrm{CF}$. These figures serve to highlight the relative advantage and disadvantage of hybrid relay configurations at various transmission rates (multiplexing gains).

\section{CONCLUSion}

This paper calculates the high-SNR performance for relay selection in a heterogeneous multi-relay network where relays of different types coexist. A variety of relay combinations with orthogonal and non-orthogonal protocols are studied. A key feature of this work is that it accounts for the presence of a direct source-destination link, which is often ignored in the relay selection literature. The results produce several qualitative insights about relay selection.

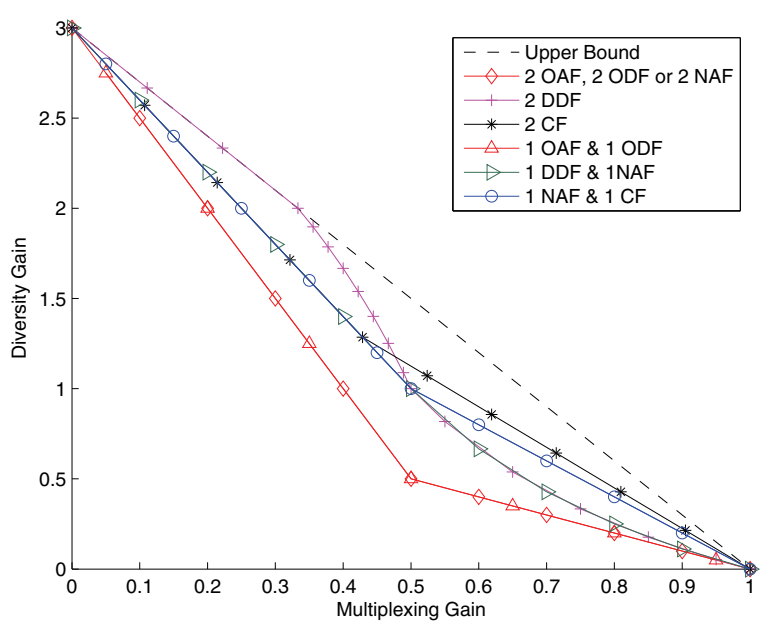

Fig. 4. The DMT of hybrid relay selection from among two relays

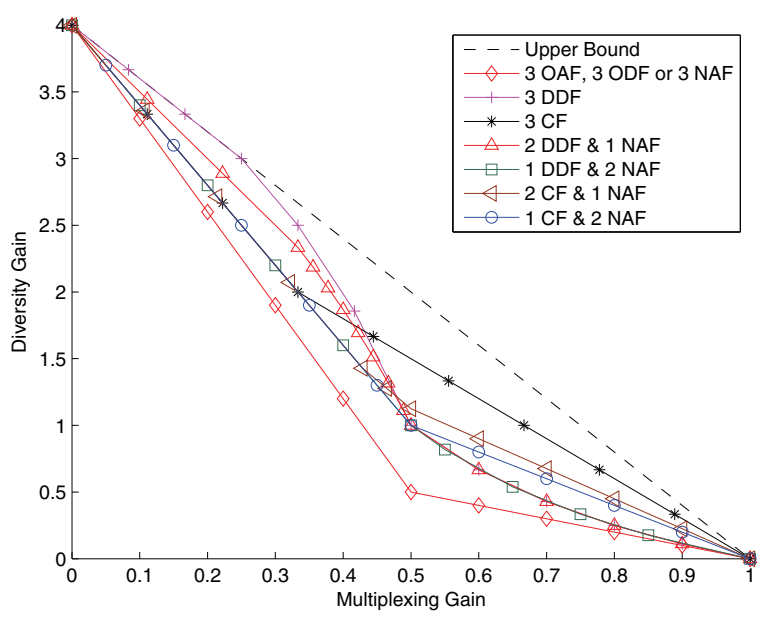

Fig. 5. The DMT of hybrid relay selection from among three relays

\section{APPENDIX}

Define $Z=X+Y$, where $X$ and $Y$ are exponential random variables with exponential parameters $\lambda_{1}$ and $\lambda_{2}$, respectively. Conditioned on the event $B=\{Y<\alpha\}$, the conditional pdf of $Y$ is given by

$$
f_{Y \mid B}(y)= \begin{cases}\lambda_{2} \frac{e^{-\lambda_{2} y}}{1-e^{-\lambda_{2} \alpha}} & y \leq \alpha \\ 0 & y>\alpha .\end{cases}
$$

The pdf of $Z=X+Y$ conditioned on the event $B$ is as follows.

$$
f_{Z \mid B}(z)= \begin{cases}\int_{0}^{z} \lambda_{1} e^{-\lambda_{1}(z-x)} \frac{\lambda_{2} e^{-\lambda_{2} x}}{1-e^{-\lambda_{2} \alpha}} d x & z \leq \alpha \\ \int_{0}^{\alpha} \lambda_{1} e^{-\lambda_{1}(z-x)} \frac{\lambda_{2} e^{-\lambda_{2} x}}{1-e^{-\lambda_{2} \alpha}} d x & z>\alpha .\end{cases}
$$




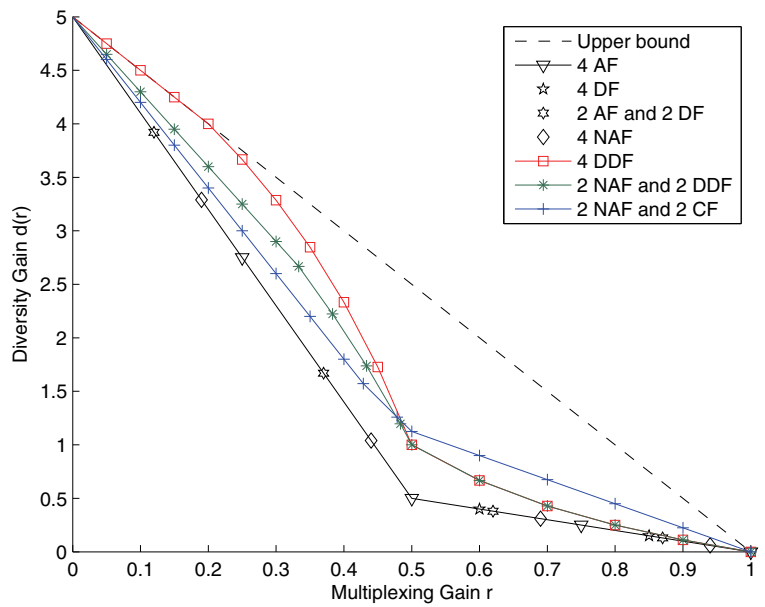

Fig. 6. The DMT of hybrid relay selection from among four relays

Hence, if $\lambda_{1} \neq \lambda_{2}$, the conditional pdf of $Z=X+Y$ is

$$
f_{Z \mid B}(z)= \begin{cases}\lambda_{1} \lambda_{2} \frac{e^{-\lambda_{1} z}\left(e^{\left(\lambda_{1}-\lambda_{2}\right) z}-1\right)}{\left(\lambda_{1}-\lambda_{2}\right)\left(1-e^{-\lambda_{2} \alpha}\right)} & z \leq \alpha \\ \lambda_{1} \lambda_{2} \frac{e^{-\lambda_{1} z}\left(e^{\left(\lambda_{1}-\lambda_{2}\right) \alpha}-1\right)}{\left(\lambda_{1}-\lambda_{2}\right)\left(1-e^{-\lambda_{2} \alpha}\right)} & z>\alpha .\end{cases}
$$

If $\lambda_{1}=\lambda_{2}=\lambda$, the conditional pdf of $Z$ is

$$
f_{Z \mid B}(z)= \begin{cases}\lambda^{2} \frac{z e^{-\lambda z}}{\left(1-e^{-\lambda \alpha}\right)} & z \leq \alpha \\ \lambda^{2} \frac{\alpha e^{-\lambda z}}{\left(1-e^{-\lambda \alpha}\right)} & z>\alpha .\end{cases}
$$

\section{REFERENCES}

[1] T. E. Hunter and A. Nosratinia, "Distributed protocols for user cooperation in multi-user wireless networks," in Proc. 2004 IEEE Global Telecommun. Conf., vol. 6, pp. 3788-3792.

[2] A. Bletsas, A. Khisti, D. P. Reed, and A. Lippman, "A simple cooperative diversity method based on network path selection," IEEE J. Sel. Areas Commun., vol. 24, no. 3, pp. 659-672, Mar. 2006.

[3] Y. Jing and H. Jafarkhani, "Single and multiple relay selection schemes and their achievable diversity orders," IEEE Trans. Wireless Commun., vol. 8, no. 3, pp. 1414-1423, Mar. 2009.

[4] I. Krikidis, J. Thompson, S. McLaughlin, and N. Goertz, "Amplifyand-forward with partial relay selection," IEEE Commun. Lett., vol. 12 , no. 4, pp. 235-237, Apr. 2008.

[5] I. Krikidis, J. Thompson, S. Mclaughlin, and N. Goertz, "Max-min relay selection for legacy amplify-and-forward systems with interference," IEEE Trans. Wireless Commun., vol. 8, no. 6, pp. 3016-3027, June 2009.

[6] M. M. Fareed and M. Uysal, "On relay selection for decode-and-forward relaying," IEEE Trans. Wireless Commun., vol. 8, no. 7, pp. 3341-3346, July 2009.

[7] A. Nosratinia and T. E. Hunter, "Grouping and partner selection in cooperative wireless networks," IEEE J. Sel. Areas Commun., vol. 25, no. 2, pp. 369-378, Feb. 2007

[8] S. Lee, M. Han, and D. Hong, "Average SNR and ergodic capacity analysis for opportunistic DF relaying with outage over Rayleigh fading channels," IEEE Trans. Wireless Commun., vol. 8, no. 6, pp. 2807-2812, June 2009.

[9] R. Tannious and A. Nosratinia, "Spectrally-efficient relay selection with limited feedback," IEEE J. Sel. Areas Commun., vol. 26, no. 8, pp. 1419-1428, Aug. 2008.

[10] R. Vaze and R. W. Heath, "To code in space and time or not in multihop relay channels," IEEE Trans. Signal Process., vol. 57, no. 7, pp. 27362747, July 2009.

[11] Y. Li, B. Vucetic, Z. Chen, and J. Yuan, "An improved relay selection scheme with hybrid relaying protocols," in Proc. 2007 IEEE Global Telecommun. Conf., pp. 3704-3708.

[12] J. Fricke, M. Butt, and P. Hoeher, "Quality-oriented adaptive forwarding for wireless relaying," IEEE Commun. Lett., vol. 12, no. 3, pp. 200-202, Mar. 2008.
[13] T. Liu, L. Song, Y. Li, Q. Huo, and B. Jiao, "Performance analysis of hybrid relay selection in cooperative wireless systems," IEEE Trans. Commun., vol. 60, no. 3, pp. 779-788, Mar. 2012.

[14] X. Bao and J. Li, "Efficient message relaying for wireless user cooperation: decode-amplify-forward (DAF) and hybrid DAF and codedcooperation," IEEE Trans. Wireless Commun., vol. 6, no. 11, pp. 39753984, Nov. 2007.

[15] M. Jeong, S. Hwang, D. Kim, and J. Yang, "Performance analysis of cooperative communication with heterogeneous relays," in Proc. 2011 IEEE Personal Indoor Mobile Radio Commun., pp. 1104-1108.

[16] P. Lusina, R. Schober, and L. Lampe, "Diversity-multiplexing trade-off of the hybrid non-orthogonal amplify-decode and forward protocol," in Proc. 2008 IEEE International Symp. Inf. Theory, pp. 2375-2379.

[17] S. Yang and J.-C. Belfiore, "Towards the optimal amplify-and-forward cooperative diversity scheme," IEEE Trans. Inf. Theory, vol. 53, no. 9, pp. 3114-3126, Sept. 2007.

[18] L. Zheng and D. N. C. Tse, "Diversity and multiplexing: a fundamental tradeoff in multiple-antenna channels," IEEE Trans. Inf. Theory, vol. 49, no. 5, pp. 1073-1096, May 2003.

[19] M. Abouelseoud and A. Nosratinia, "Opportunistic wireless relay networks: diversity-multiplexing tradeoff," IEEE Trans. Inf. Theory, vol. 57, no. 10 , pp. 6514-6538, Oct. 2011.

[20] J. Laneman, D. Tse, and G. Wornell, "Cooperative diversity in wireless networks: efficient protocols and outage behavior," IEEE Trans. Inf. Theory, vol. 50, no. 12, pp. 3062-3080, Dec. 2004.

[21] M. O. Hasna and M. S. Alouini, "End-to-end performance of transmission systems with relays over Rayleigh-Fading channels," IEEE Trans. Wireless Commun., vol. 2, no. 6, pp. 1126-1131, Nov. 2003.

[22] K. Seddik, A. Sadek, W. Su, and K. Liu, "Outage analysis of multi-node amplify-and-forward relay networks," in Proc. 2006 Wireless Commun. Netw. Conf., pp. 1184-1188.

[23] Y. Jiang and M. Varanasi, "Diversity-multiplexing tradeoff of MIMO system with antenna selection," in Proc. 2007 IEEE International Symp. Inf. Theory, pp. 2836-2840.

[24] R. U. Nabar, H. Bolcskei, and F. W. Kneubuhler, "Fading relay channels: performance limits and space-time signal design," IEEE J. Sel. Areas Commun., vol. 22, no. 6, pp. 1099-1109, Aug. 2004.

[25] K. Azarian, H. El Gamal, and P. Schniter, "On the achievable diversitymultiplexing tradeoff in half-duplex cooperative channels," IEEE Trans. Inf. Theory, vol. 51, no. 12, pp. 4152-4172, Dec. 2005.

[26] M. Yuksel and E. Erkip, "Multiple-antenna cooperative wireless systems: a diversity multiplexing tradeoff perspective," IEEE Trans. Inf. Theory, vol. 53, no. 10, pp. 3371-3393, Oct. 2007.

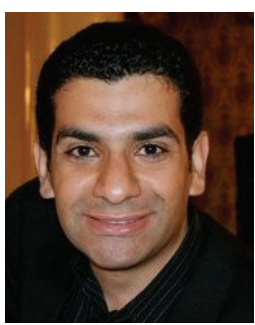

Mohamed Abouelseoud (S'09) received the B.S. in electrical engineering and the M.S. in Engineering Mathematics from Cairo University, Egypt, in 2003 and 2006, respectively. Currently, he is working toward the Ph.D. degree in electrical engineering at the University of Texas at Dallas. His research interests lie in the broad area of wireless communication and information theory, with emphasis on cooperative communications and relay networks. Since May 2010, he has been with InterDigital Communications, Inc., King of Prussia, PA, as a Senior Systems Engineer in the Advanced Air Interface Group. He is involved in research on the next generation wireless cellular systems such as LTE-A and beyond.

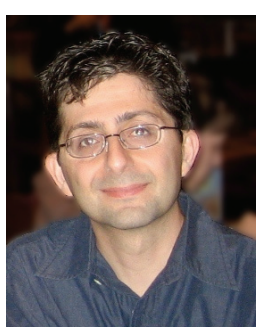

Aria Nosratinia (S'87-M'97-SM'04-F'10) is Erik Jonsson Distinguished Professor and associate head of the Electrical Engineering Department at the University of Texas at Dallas. He received his Ph.D. in Electrical and Computer Engineering from the University of Illinois at Urbana-Champaign in 1996. He has held visiting appointments at Princeton University, Rice University, and UCLA. His interests lie in the broad area of information theory and signal processing, with applications in wireless communications. He was the secretary of the IEEE Information Theory Society in 2010-2011 and the treasurer for ISIT 2010 in Austin, Texas. He has served as editor for the IEEE TRANSACTIONS ON INFORMATION THEORY, IEEE TRANSACTIONS ON WIRELESS COMMUNICATIONS, IEEE SignAL PROCESSING LETTERS, IEEE TRANS ACTIONS ON IMAGE Processing, and IEEE Wireless Communications Magazine. He has been the recipient of the National Science Foundation career award, and is a fellow of IEEE. 